\title{
AIR KERMA TO HP(3) CONVERSION COEFFICIENTS FOR IEC 61267 RQR X-RAY RADIATION QUALITIES. APPLICATION TO DOSE MONITORING OF THE LENS OF THE EYE IN MEDICAL DIAGNOSTICS
}

\author{
S. Principi, C. Guardiola, M.A. Duch, M. Ginjaume \\ Institut de Tècniques Energètiques. Universitat Politècnica de Catalunya (UPC). 08028 Barcelona. Spain.
}

\begin{abstract}
Recent studies highlight the fact that the new eye lens dose limit can be exceeded in interventional radiology procedures and that eye lens monitoring could be required for these workers. The recommended operational quantity for monitoring of eye lens exposure is the personal dose equivalent at $3 \mathrm{~mm}$ depth $H_{\mathrm{p}}(3)$ (ICRU 51). However, there are no available conversion coefficients in international standards, while in the literature coefficients have only been calculated for mono-energetic beams and for ISO 4037-1 X-ray qualities.

The aim of this paper is to provide air kerma to $H_{\mathrm{p}}(3)$ conversion coefficients for a cylindrical phantom made of ICRU-4 elements tissue-equivalent material for RQR radiation qualities (IEC-61267) from $40 \mathrm{kV}$ to $120 \mathrm{kV}$ and for angles of incidence from $\mathbf{0}^{\circ}$ to $\mathbf{1 8 0}^{\circ}$, which are characteristic of medical workplaces. Analytic calculations using interpolation techniques and Monte Carlo modeling have been compared.
\end{abstract}

\section{INTRODUCTION}

The recommended operational quantity to monitor exposure to the eye lens is the personal dose equivalent at $3 \mathrm{~mm}$ depth $H_{\mathrm{p}}(3)^{(1)}$. However, in practice, this quantity has rarely been used up to now. International standard IEC 62387:2012 $2^{(2)}$ does not provide an appropriate phantom for calibrating personal dosemeters to be worn on the head, and consequently neither conversion coefficients for this purpose. Several works have recently supplied complementary data and proposals to improve the assessment of the dose equivalent to the eye lens. Within the framework of the European project ORAMED ${ }^{(3)}$, Gualdrini et al. ${ }^{(4)}$ recommended the use of an ICRU-4 element tissue equivalent cylindrical phantom of $20 \mathrm{~cm}$ diameter and $20 \mathrm{~cm}$ height. This proposal was based on anatomical considerations, since this geometry is closer to the mass and shape of the head than the $30 \times 30 \times 15 \mathrm{~cm}^{3}$ ISO slab phantom $^{(5)}$. The authors ${ }^{(6)}$ also provided conversion coefficients from air kerma to $H_{\mathrm{p}}(3)$ for mono-energetic photon fields and for some X-ray beams of interest. Behrens (2010) published a complete series of $h_{\mathrm{pK}}(3, \mathrm{R}, \alpha)$ for ISO 4037 $1^{(7)}$ qualities using a slab phantom and later on in 2012 he published a series for the cylindrical phantom ${ }^{(8,9)}$. The recent interest and need to perform eye lens monitoring is due to the new recommendations of ICRP $118^{(10)}$ of a dose limit of $20 \mathrm{mSv}$ per year for occupational exposure to the eye. The new requirement is a serious challenge especially for the medical fields of interventional radiology and cardiology. The aim of this paper is to provide air kerma to $H_{\mathrm{p}}(3)$ conversion coefficients for the cylindrical phantom for RQR IEC-61267 ${ }^{(11)} \mathrm{X}$-ray beams for angles of incidence from $0^{\circ}$ to $180^{\circ}$. The RQR2 to RQR9 IEC-61267 qualities were chosen because they provide a better approximation of the radiation spectra found in practice in interventional cardiology and radiology workplaces than ISO 4037-1 qualities and are often used in intercomparisons in this field. The study also discusses the influence of different approaches used in the literature for the calculation of conversion coefficients. As opposed to ISO 4037-1 qualities, IEC-61267 does not specify the filtration needed to produce RQR beams. RQR qualities are defined by the tube voltage and the nominal first half-value layer. In order to be reproduced, one has to adjust them in order to obtain a ratio between air kerma (or air kerma rate) with and without a filter of thickness equal to the nominal first half-value layer between 0.485 and 0.515 . The study estimates the influence of the filtration used in different laboratories for $h_{\mathrm{pK}}(3)$ conversion coefficients.

\section{MATERIALS AND METHODS}

The kerma-to-personal dose equivalent $H_{\mathrm{p}}(3)$ conversion coefficient for the cylinder is defined as the ratio of the quantities $H_{\mathrm{p}}(3)$ and the air kerma $K_{\mathrm{a}}$ :

$$
h_{\mathrm{p} K}(3, \mathrm{RQR}, \alpha)_{\mathrm{cyl}}=H_{\mathrm{p}}(3) / K_{\mathrm{a}}
$$

The conversion coefficient depends on the energy, the directional distribution of the incident radiation and also the phantom used in the calibration. $h_{\mathrm{pK}}(3, \mathrm{RQR}, \alpha)_{\mathrm{cyl}}$ values for different RQR radiation qualities and angles $\alpha$ from 0 to $180^{\circ}$ were assessed by means of two methods: analytic calculations through interpolation techniques and Monte Carlo modeling. Both methods are detailed below. As mentioned above, RQR qualities can be generated by using different added filtration and, therefore, the energy spectrum of the beams can be slightly different depending on the laboratory. In this paper calculations are performed for the RQR spectra generated at our secondary standard

*Corresponding author: sara.principi@upc.edu 
laboratory at Universitat Politècnica de Catalunya (UPC) (column 3 in Table 1). To evaluate the influence of the filtration used by different laboratories, calculations are repeated for the RQR spectra used by the Metrology Institute of Germany Physikalisch-Technische Bundesanstalt (PTB), using the information provided in PTB calibration certificates (column 4 in Table 1). The inherent filtration for UPC and PTB beams is equal to $7 \mathrm{mmBe}$. In addition, results have also been compared to $h_{\mathrm{p} K}(3)$ coefficients calculated by the French national metrology Laboratory CEA LIST/LNE LNHB ${ }^{(3)}$ for RQR7 and RQR9 nominal HVL values (column 5, Table 1).

\section{Monte Carlo simulation}

The simulation study to obtain the conversion coefficients was performed using two Monte Carlo codes: PENELOPE $^{(13)}$ and MCNPX ${ }^{(14)}$.

The air kerma and $H_{\mathrm{p}}(3)$ in the ICRU tissue cylindrical phantom were calculated employing both Monte Carlo codes to obtain the $h_{\mathrm{p} K}(3, \mathrm{RQR}, 0)$ conversion factors for normal incidence. Although $H_{\mathrm{p}}(3)$ and $K_{\mathrm{a}}$ are obtained per unit fluence, i.e. $H_{\mathrm{p}}(3) / \Phi$ and $K_{\mathrm{a}} / \Phi$, they are referred to as $H_{\mathrm{p}}(3)$ and $K_{\mathrm{a}}$ for simplicity. In the set-up geometry the cylindrical phantom surrounded by air is irradiated by a $20 \mathrm{~cm}$ x $20 \mathrm{~cm}$ collimated square beam, placed at $1 \mathrm{~m}$ from the phantom front-face. To assess $H_{\mathrm{p}}(3)$ values, a 0.5 -mm-thick sensitive volume was placed at $3 \mathrm{~mm}$ depth within the cylinder. Parallelepipeds of $1 \mathrm{~mm}$ width, $0.5 \mathrm{~mm}$ thickness, and $5 \mathrm{~cm}$ height were used as scoring volumes for both Monte Carlo codes. Conversion coefficients for angles from 0 to 180 degrees were evaluated only with MCNPX. PENELOPE was used to compare the results only for normal incidence. The statistical uncertainty of the Monte Carlo simulations was within 0.1-1 \% at one standard deviation. It is worth mentioning that PENELOPE and MCNPX manage the simulation output process in different ways.

The requested PENELOPE output for the calculation of $H_{\mathrm{p}}(3)$ was the energy deposited in the detection material. This quantity was then divided by the mass of the scoring volume to obtain the absorbed dose at $3 \mathrm{~mm}$ depth. Cut-off energies were set to $1 \mathrm{keV}$ both for electrons and positrons. On the other hand, for the calculation of the air kerma, transport of secondary electrons and positrons was disregarded by setting both cut-off energies equal to the maximum energy of the radiation spectrum, which is the voltage applied to the X-ray tube.

For the MCNPX calculations, energy deposition tally F6 was used. hpk(3,RQR,0) coefficients obtained by PENELOPE were calculated considering the secondary electron transport, while those obtained by MCNPX disregarded the secondary particles (kerma approximation mode). $3 \mathrm{~mm}$ is the maximum range of electrons generated by $1 \mathrm{MeV}$ photons in ICRU tissue; thus this comparison is aimed on one hand to verify the validity of the air kerma approximation and on the other to evaluate the differences when using two well-known MC programmes.

\section{Analytical method}

$h_{\mathrm{pK}}(3, \mathrm{RQR}, 0)$ conversion coefficients were calculated for RQR qualities and for a varying angle of incidence from 0 to $180^{\circ}$ by implementing the interpolation technique suggested by Behrens ${ }^{(8)}$. Conversion coefficients for monoenergetic photon beams were taken from Gualdrini et al. ${ }^{(6)}$. The following steps were considered:

(a) Firstly, the photon fluence spectra $(\mathrm{d} \varphi / \mathrm{dE})$ for the radiation qualities of interest were determined from the XCOMP5 program ${ }^{(15)}$. The fluence values per unit energy are given for integer values of energy from $1 \mathrm{keV}$ to the tube voltage $\left(\mathrm{V}_{\max }\right)$, in steps of $1 \mathrm{keV}$. The input data introduced for each quality and laboratory were: tube voltage, inherent filtration (7 mmBe), additional filtration and anode angle of the tube $\left(18^{\circ}\right)$.

(b) Subsequently, the average conversion coefficients $h_{\mathrm{pK}}(3, \mathrm{RQR}, \alpha)$ were calculated by applying the following equation:

$$
h p k(3, R Q R, \alpha)=\frac{\sum_{i=1 \mathrm{keV}}^{V \max } \frac{d \varphi}{d E} \cdot h p k\left(3, E_{i}, \alpha\right) \cdot E_{i} \cdot \frac{\mu_{e n}\left(E_{i}\right)}{\rho}}{\sum_{i=1 k e V}^{V \max } \frac{d \varphi}{d E} \cdot E_{i} \cdot \frac{\mu_{e n}\left(E_{i}\right)}{\rho}}
$$

$h_{\mathrm{pK}}(3, \mathrm{Ei}, \alpha)$ correspond to mono-energetic photons for the ICRU cylindrical phantom calculated by Gualdrini et al. ${ }^{(6)}$

The formula represents the ratio between dose equivalent at $3 \mathrm{~mm}$ depth and air kerma, calculated for the radiation quality of interest RQR. In the equation, $d \varphi / d E$ is the fluence per unit energy and Vmax is the voltage applied to the $\mathrm{X}$-ray tube to generate the radiation beam. $h_{\mathrm{p} K}(3, \mathrm{RQR}, \alpha)$ are the conversion coefficients obtained by using a cubic spline interpolation at low energies (for energies between $10 \mathrm{keV}$ and $40 \mathrm{keV}$ and for angles larger than $90^{\circ}$ ) and a linear-logarithmic interpolation (linear in values and logarithmic in energy). The mass-energy absorption coefficients $\mu_{\mathrm{en}}\left(\mathrm{E}_{\mathrm{i}}\right) / \rho$ for photons in air are obtained by log-log interpolation (logarithmic both in energy and in values) from available values taken from Hubbell ${ }^{(16)}$. 


\section{Monte Carlo simulation}

The difference between PENELOPE and MCNPX $h_{\mathrm{pK}}(3, \mathrm{RQR}, 0)$ were below $1 \%$. The statistical uncertainty for both MC codes was within $1 \%$, for one standard deviation. This result confirms the validity of the kerma approximation used in the following calculations.

\section{Analytical method}

The MCNPX output was compared to the values obtained by interpolation. For all the considered incident angles $h_{\mathrm{pK}}(3, \mathrm{RQR}, \alpha)$ differences were lower than $0.8 \%$. This result highlights the fact that the analytical method is both a good and quick estimation tool for the calculation of conversion coefficients within the analyzed energy range provided you have the conversion coefficients for mono energetic photon beams. As suggested by Behrens, for angles larger than $90^{\circ}$ an approximation with a cubic polynomial can better estimate conversion coefficients at low energies from 10 up to $40 \mathrm{keV}$, and thus avoid unrealistic results. Indeed, in this angle and energy range, the difference between analytical and simulated outputs is reduced from a maximum of $7 \%$ (linear interpolation on a log-lin) to values within $0.8 \%$ (cubic spline interpolation). Therefore, Monte Carlo modeling has been considered the golden standard method even if interpolation results lead to very good approximations when this technique has been chosen carefully.

\section{Influence of RQR reproduced in different laboratories}

Table 2 data were compared with $h_{\mathrm{pK}}(3, \mathrm{RQR}(\mathrm{PTB}), \alpha)$ for RQR qualities from PTB, this is with the filtration indicated in Table 1 column 4, calculated with the analytical method. Results showed good agreement. Differences were below $1.5 \%$ for angles smaller than $90^{\circ}$ and between 1 and $7 \%$ for larger angles. The larger disagreement was found for RQR5 and RQR8 qualities, where differences in added filtration are higher.

In addition, Table 2 data were also compared with the conversion coefficients published in Table 2.7 in ORAMED report ${ }^{(4)}$ for RQR7 and RQR9, for angles up to $90^{\circ}$. In this case, results agreed to within $0.6 \%$.

\section{CONCLUSIONS}

Conversion coefficients from air kerma to equivalent dose at $3 \mathrm{~mm}$ depth for radiation qualities RQR 5 to 9 and for angles of incidence from 0 to $180^{\circ}$ are provided for the cylindrical phantom. The given data were calculated by using the MCNPX Monte Carlo code in the kerma approximation and were validated for normal incidence by using the PENELOPE Monte Carlo code with secondary particle transport. Although Monte Carlo calculations were considered the golden standard method, the study demonstrates the utility of the interpolation method to calculate specific conversion coefficients when this technique is chosen carefully. The conversion coefficients given in Table 2 are calculated for RQR qualities as defined in our lab. However, it has been verified that up to an angle of incidence of $90^{\circ}$ they can be used by other laboratories to within an uncertainty of $2 \%$ (one standard deviation). This is the same uncertainty stated in ISO 4037-3 for ISO 4037-1 qualities conversion coefficients.

\section{FUNDING}

This work was supported by the Spanish National Council in the framework of the 2012-2014 call for projects on Radiological Protection.

\section{REFERENCES}

1. International Commission on Radiation Units and Measurements. Quantities and Units in Radiation Protection Dosimetry. ICRU Report 51. ICRU (1993).

2. IEC 62387. Radiation protection instrumentation - Passive integrating dosimetry systems for personal and environmental monitoring of photon and beta radiation (2012).

3. Vanhavere, F., Carinou, E., Gualdrini, G., Clairand, I., Sans Merce, M., Ginjaume, M., Nikodemova, D., Jankowski, J., Bordy, J-M., Rimpler, A., Wach, S., Martin, P., Struelens, L., Krim, S., Koukorava, C., Ferrari, P., Mariotti, F., Fantuzzi, E., Donadille, L., Itié, C., Ruiz, N., Carnicer, A., Fulop, M., Domienik, J., Brodecki, M., Daures, J., Barth, I., Bilski, P. ORAMED: Optimization of Radiation Protection of Medical Staff. EURADOS Report 2012-02, ISSN 2226-8057, ISBN 978-3-943701-012, pg. 88. Braunschweig (2012).

4. Gualdrini, G., Mariotti, F., Wach, S., Bilski, P., Denoziere, M., Daures, J., Bordy, J.-M., Ferrari, P., Monteventi, F., Fantuzzi, E., Vanhavere F. A new cylindrical phantom for eye lens dosimetry development. Radiation Measurements 46(11), 1231-1234 (2011). 
5. International Organization for Standardization. $\mathrm{X}$ and gamma reference radiation for calibrating dosemeters and dose rate meters and for determining their response as a function of photon energy-Part 3: Calibration of area and personal dosemeters and the measurement of their response as a function of energy and angle of incidence. ISO 4037-3. ISO (1999).

6. Gualdrini,G.; Bordy, J. M.; Daures J.; Fantuzzi, E.; Ferrari, P.; Mariotti, F.; Vanhavere, F. Air kerma to Hp(3) conversion coefficients for photons from $10 \mathrm{keV}$ to $10 \mathrm{MeV}$, calculated in a cylindrical phantom. Radiat. Prot. Dosim, 154 (4): $517-521$ (2013).

7. International Organization for Standardization. $\mathrm{X}$ and gamma reference radiation for calibrating dosemeters and dose rate meters and for determining their response as a function of photon energy-Part 1: Radiation characteristics and production method. ISO 4037-1. ISO (1996).

8. Behrens, R. Air kerma to dose equivalent conversion coefficients not included in ISO 4037-3. Radiat. Prot. Dosim, 147(3):3739 (2011).

9. Behrens, R. Air kerma to dose equivalent conversion coefficients for a new cylinder phantom for photon reference radiation qualities. Radiat. Prot. Dosim, 151(3):450-5, 2012.

10. International Commission on Radiological Protection. ICRP Statement on tissue reactions / early and late effects of radiation in normal tissues and organs - threshold doses for tissue reactions in a radiation protection context. ICRP Publication 118. Ann. ICRP 41(1/2) (2012).

11. International electro technical commission (IEC) Medical diagnostic X-ray equipment-radiation conditions for use in the determination of characteristics. 61267 Ed. 2.0. IEC (2005)

12. PTB calibration certificate dated 2012-06-20. Calibration mark PTB60188-12.

13. Salvat F., Fernández-Varea J.M., Sempau J., 2006. PENELOPE 2006: A code system for Monte Carlo simulation of electron and photon transport. Workshop Proceedings Barcelona, Spain, 4-7 July 2006, OECD 2006 NEA No. 6222, Nuclear Energy Agency Organisation for Economic Co-operation and Development.

14. Pelowitz, D.B. (Ed.), 2005. MCNPX User's Manual, Version 2.6.0. Los Alamos National Laboratory Report LA-CP-05-0369.

15. Nowotny, R., \& Hofer, A. (1985). XCOMP5, Program for calculating diagnostic X-ray spectra. RoeFo, Fortschr. Geb.Roentgenstr. Nuklearmed. 142, pp. 685-689.

16. Hubbell, J. H. and Seltzer, S. M. Tables of X-ray mass attenuation coefficients and mass energy-absorption coefficients 1 keV to $20 \mathrm{MeV}$ for elements $\mathrm{Z}=1$ to 92 and 48 additional substances of dosimetric interest. Report NISTIR 5632 (1995). The data are available on http://www.nist.gov/pml/data/xraycoef/index.cfm. 
Table 1. Characteristics of RQR qualities used in this paper for the $h_{p k}(3, R Q R, \alpha)_{\text {cyl }}$ calculation Tube HVL (mmAl) Added filtration (mmAl)

voltag UPC PTB* CEA $^{* *}$ UPC PTB CEA $(\mathrm{kV})$

\begin{tabular}{|c|c|c|c|c|c|c|c|}
\hline RQR2 & 40 & 1.41 & 1.42 & & 2.5 & 2.49 & \\
\hline RQR3 & 50 & 1.78 & 1.77 & & 2.5 & 2.46 & \\
\hline RQR4 & 60 & 2.11 & 2.19 & & 2.5 & 2.68 & \\
\hline RQR5 & 70 & 2.39 & 2.57 & & 2.5 & 2.83 & \\
\hline RQR6 & 80 & 3.01 & 3.01 & & 3.0 & 2.99 & \\
\hline RQR7 & 90 & 3.48 & 3.48 & 3.48 & 3.0 & 3.18 & 3.0 \\
\hline RQR8 & 100 & 3.86 & 3.96 & & 3.0 & 3.36 & \\
\hline RQR9 & 120 & 4.98 & 5.00 & 5.00 & 3.5 & 3.73 & 3.39 \\
\hline
\end{tabular}

* Data obtained from PTB calibration certificates ${ }^{(12)}$ ** CEA LIST/LNE LNHB Table 2.6 reference (3) 
Table 2. Conversion coefficients $h_{p k}(3, R Q R, \alpha)_{c y l}$ from air kerma to dose equivalent at $3 \mathbf{m m}$ depth for $R Q R$ qualities and for angles of incidence from 0 to $180^{\circ}$.

$\operatorname{hpk}(3, R, \alpha)$ - MCNPX

\begin{tabular}{ccccccccc}
\hline Angles & RQR2 & RQR3 & RQR4 & RQR5 & RQR6 & RQR7 & RQR8 & RQR9 \\
$\mathbf{0}^{\circ}$ & 1.106 & 1.178 & 1.232 & 1.27 & 1.336 & 1.368 & 1.394 & 1.456 \\
$\mathbf{1 0}^{\circ}$ & 1.099 & 1.172 & 1.226 & 1.268 & 1.337 & 1.369 & 1.396 & 1.455 \\
$\mathbf{1 5}^{\circ}$ & 1.099 & 1.172 & 1.226 & 1.269 & 1.336 & 1.369 & 1.397 & 1.456 \\
$\mathbf{2 0}^{\circ}$ & 1.094 & 1.167 & 1.221 & 1.262 & 1.329 & 1.363 & 1.39 & 1.449 \\
$\mathbf{3 0}^{\circ}$ & 1.081 & 1.154 & 1.208 & 1.247 & 1.314 & 1.347 & 1.373 & 1.437 \\
$\mathbf{4 5}^{\circ}$ & 1.043 & 1.117 & 1.171 & 1.215 & 1.283 & 1.317 & 1.346 & 1.408 \\
$\mathbf{6 0}^{\circ}$ & 0.965 & 1.041 & 1.097 & 1.141 & 1.211 & 1.247 & 1.276 & 1.341 \\
$\mathbf{7 5}^{\circ}$ & 0.800 & 0.882 & 0.941 & 0.989 & 1.064 & 1.102 & 1.134 & 1.207 \\
$\mathbf{9 0}^{\circ}$ & 0.450 & 0.533 & 0.595 & 0.643 & 0.723 & 0.766 & 0.802 & 0.884 \\
$\mathbf{1 0 5}^{\circ}$ & 0.131 & 0.186 & 0.232 & 0.269 & 0.33 & 0.365 & 0.396 & 0.467 \\
$\mathbf{1 2 0}^{\circ}$ & 0.039 & 0.067 & 0.093 & 0.117 & 0.153 & 0.177 & 0.198 & 0.246 \\
$\mathbf{1 3 5}^{\circ}$ & 0.015 & 0.030 & 0.046 & 0.062 & 0.086 & 0.102 & 0.117 & 0.151 \\
$\mathbf{1 5 0}^{\circ}$ & 0.008 & 0.018 & 0.029 & 0.04 & 0.057 & 0.07 & 0.081 & 0.107 \\
$\mathbf{1 6 5}^{\circ}$ & 0.005 & 0.013 & 0.022 & 0.031 & 0.046 & 0.056 & 0.066 & 0.088 \\
$\mathbf{1 8 0}^{\circ}$ & 0.004 & 0.011 & 0.020 & 0.029 & 0.042 & 0.052 & 0.062 & 0.082 \\
\hline & & & & & & & &
\end{tabular}

